\section{Population genomics: celebrating individual expression}

\author{
G Gibson
}

Heredity (2003) 90, 1-2. doi:10.1038/sj.hdy.6800195

$\mathrm{F}$ uture efforts to map genotype onto phenotype may well use gene expression profiling alongside scanning for association between genetic variants and traits. The basic idea is similar, but the difference is that we will look for transcripts rather than SNPs that are correlated with a phenotype. In fact, microarray analysis offers a couple of advantages for quantitative genetic analysis. One is that it is efficient and comprehensive, so true genome-wide scans can be envisaged for a wide variety of organisms. The other is that transcript abundance already integrates developmental and environmental noise with regulatory polymorphisms. Thus, we might expect variation in transcript abundance to be somewhat closer to phenotypic variation than are the actual genotypes.

Oleksiak et al (2002) new landmark study in empirical population genomics is the first to use cDNA microarrays to compare individuals within and among populations. They examined transcript levels of 907 genes from heart ventricle tissue of 15 adult Fundulus fish from three different populations, all raised in the same controlled environment.
Fundulus is the genus of minnows that are famous in evolutionary genetic circles as a model for physiological adaptation to temperature (Podrabsky et al, 2000). The cline of activity of the $L d h$ enzyme (Crawford and Powers, 1989) is particularly well characterized, and Crawford's group has now begun to ask just how widespread adaptation may be at the gene expression level.

The first conclusion of the study (Oleksiak et al, 2002) is that $18 \%$ of the genes in the EST collection appear to be differentially expressed among the 15 individuals, at the $1 \%$ significance level. In coming to this conclusion, they performed analysis of variance using the error associated with technical replication to assess the significance of biological variation. Each sample was measured on eight independent arrays with a loop design that ensured that the two labeling dyes, Cy3 and Cy5, were flipped and that contrasts were made among all of the populations in a balanced manner. This obviates the need for a reference sample, and results in considerable statistical power (Kerr and Churchill, 2001). Since only nine of the genes would be expected to differ among any contrasts at the $1 \%$ level by chance, 161 genes is an impressive fraction of the transcriptome. Even after correction for the 907 comparisons, 37 genes remained significant, with among-individual differences of twofold or more.

Fewer differences were detected among populations than between individuals. The power of this analysis was not the same as that for the betweenindividual comparison, but only 15 genes were seen to differ at the $1 \%$ level among the three populations. Overall, there was a trend for the genes that are most variable within populations to diverge between populations. This is expected of standard neutral theory, which reflects the common-sense intuition that things that differ more among members of a group are more likely to diverge when the group is split apart. However, this apparently banal prediction has the major implication that 'transcriptional drift' could be the predominant cause of divergence in gene expression among species.

Nevertheless, adaptation is likely to have played a major role in the divergence of the genes that showed the highest among-population divergence. Two of the populations were from one species, Fundulus heteroclitus, while the third was from another species, F. grandis, whose range overlapped with the southern population of $F$. heteroclitus. Post hoc comparisons revealed that for 27 genes, the difference in expres-

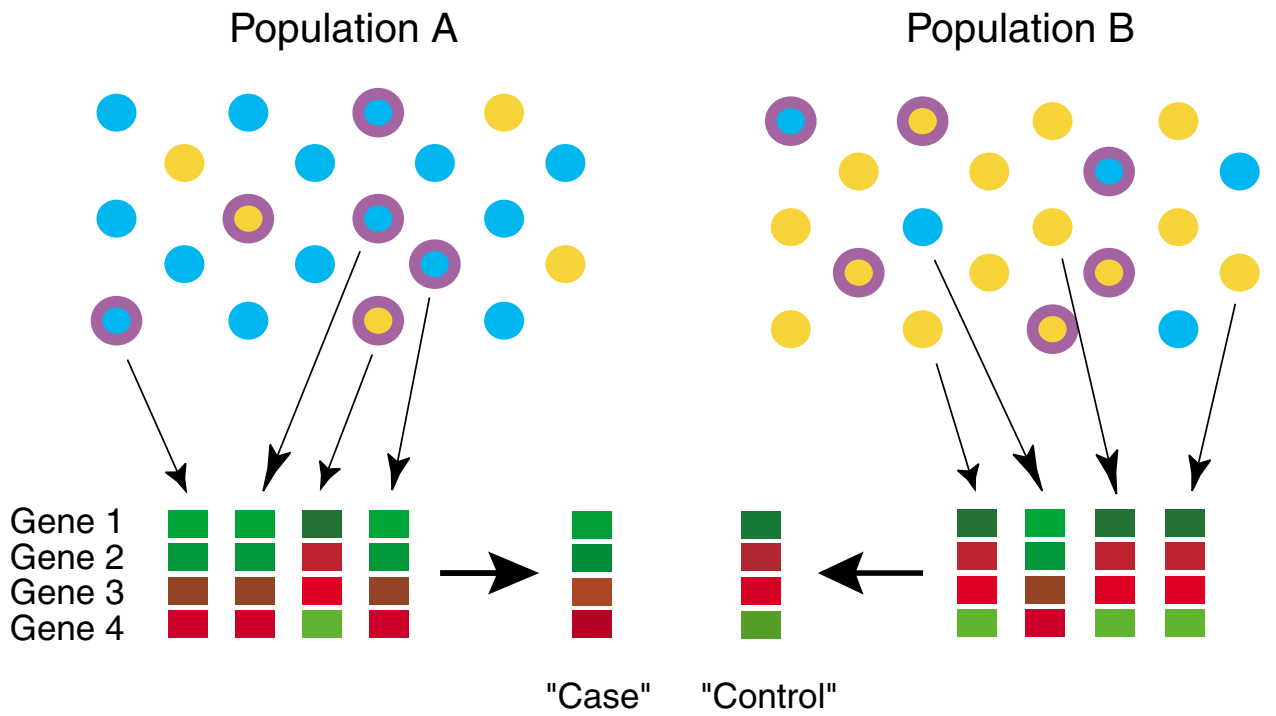

Figure 1 Potential for population stratification in gene expression profiling. Individuals with a particular disease (the 'cases', indicated by circles surrounded by a dark-blue ring) are at equal frequency in two populations. Within these populations, there are different frequencies of blue and orange individuals, who consistently differ in the expression profile of four genes, shown below. Even though there is no association between disease and expression type, random sampling of case and control individuals from structured populations could produce the spurious result indicated at the bottom, that the expression of the four genes averaged across individuals is correlated with the disease. 
sion was not so much between the species (as would be expected if expression follows overall genetic divergence), but rather between the northern population and the two southern populations. These are then good candidate genes for investigation of a role in physiological adaptation to the marine thermal gradient.

All of these results are to a broad approximation in agreement with those from several other organisms. Two recent comparisons of haploid yeast strains (Cavalieri et al, 2000; Brem et al, 2002) suggest that up to $25 \%$ of the transcriptome differs among isolates, as did a study of Drosophila melanogaster adults (Jin et al, 2001). None of these studies compared individuals, but they both showed that there is a genetic component to transcription. By contrast, the Fundulus study shows that there is among-individual variation, but not formally that this is genetic. Perhaps, the next study of this sort will allow a true estimate of transcriptional heritability by including both among-individual and among-genotype comparisons. It is also worth noting that expression profiling of primate brains and livers, as well as of these tissues in three murine species, also indicates that perhaps between 5 and $20 \%$ of the transcriptome varies among individuals (Enard et al, 2002), though comparable statistical analyses of these data have not been published.

The value of these studies from the point of view of evolutionary genetics is not difficult to see, but should genome biologists in general take note? The simple answer is yes, because it is now clear that individual variation may be confounded with experimental contrasts.

Few would argue that when contrasting gene expression profiles of patients who have a rare liver disease with those of healthy people, it is essential to ensure that the individuals are matched for age and sex. For example, if the diseased sample was from a 50-year-old woman and the control was a 21-yearold man, there may be other factors that actually cause differences in the liver expression profiles. But how careful should we be to match the individuals for ethnicity or other types of population structure?

Suppose in a clinical comparison that the population from which the controls are drawn is subtly different in some unknown manner from the population from which the cases are drawn (Figure 1). This difference could be dietary or environmental or even genetic, and the populations could be admixed. Suppose further that the difference affects the frequency of the number of individuals who share a particular pattern of altered gene expression that is actually unrelated to the disease being studied. It turns out that even if the frequency of cases is the same in the two populations, a spurious association between transcript abundance and disease status could appear, much as population structure confounds genotype association mapping (Nielsen and Zaykin, 2001). There are no easy solutions to this problem, but it at least behooves us to respect differences in individual expression.

$G$ Gibson is at the Department of Genetics, Gardner Hall, North Carolina State University, Raleigh, NC 27695-7614, USA.

e-mail: ggibson@unity.ncsu.edu

Brem RB, Yvert G, Clinton R, Kruglyak L (2002). Science 296: 752-755.

Cavalieri D, Townsend JP, Hartl DL (2000). Proc Natl Acad Sci (USA) 97: 12369-12374.

Crawford DL, Powers DA (1989). Proc Natl Acad Sci (USA) 86: 9365-9369.

Enard W, Khaitovich P, Klose J, Zöllner S, Heissig F, Giaralsson P (2002). Science 296: 340-343

Jin W, Riley R, Wolfinger R, White KP, Passador-Gurgel G, Gibson G (2001). Nat Genet 29: 389-395.

Kerr MK, Churchill GA (2001). Genet Res 77: 123-128.

Nielsen DM, Zaykin D (2001). Expert Rev Mol Diagn 1: 334-342.

Oleksiak MF, Churchill GA, Crawford DL (2002). Nat Genet 32: 261-266.

Podrabsky JB, Javillonar C, Hand S, Crawford DL (2000). Am J Physiol 279: R2344-R2348.

Comparative genomics

\section{P̈lant genomes: cyanobacterial genes revealed}

JM Archibald and PJ Keeling

Heredity (2003) 90, 2-3. doi:10.1038/sj.hdy.6800204

$\mathrm{M}$ ore than 20 years after it was proposed that plant genomes must contain genes derived from the cyanobacterial ancestor of the plastid (Weeden, 1981), the full impact of endosymbiotic gene transfer is just being revealed. In a recent study published in the Proceedings of the National Academy of Sciences, Martin et al (2002) show that the contribution of cyanobacterial genes to the nuclear genome of the flowering plant Arabidopsis extends far beyond those associated with photosynthesis or the plastid. Cyanobacterial-derived genes appear to make up a large fraction of the plant genome and they not only encode proteins that service the plastid, but proteins for all other cellular functions. These results underscore the importance of endosymbiosis in shaping the biochemistry and cell biology of eukaryotic cells.

In 1981 Weeden crystallized a major component of the theory of endosymbiosis when he proposed that plant nuclear genomes contained genes originating from the cyanobacterium that gave rise to the plastid. Weeden knew that plastids contained far more proteins than their reduced genomes could possibly encode. He suggested that these proteins were originally encoded in the endosymbiont genome, but were transferred to the host nucleus during the early stages of endosymbiosis. He also proposed that the protein products of these genes were somehow targeted to the plastid after they were translated, so that the proteins ended up where they had always been. At the time this idea was galvanizing, now it is taken for granted: hundreds of genes for plastid-targeted proteins have now been described in plant nuclei and the process by which the proteins are targeted to the plastid is now largely understood (McFadden, 1999).

While Martin et al (2002) now elucidate the full impact of endosymbiosis in shaping the plant nuclear genome, the idea that the cyanobacterial endosymbiont contributed more genes to the nucleus than strictly necessary for plastid function is not new. For example, higher plants contain two nuclear-encoded isoforms of the enzyme phosphoglycerate kinase (PGK), one functioning in the plastid, the other in the cytosol. Despite the different evolutionary histories of the cellular compartments in which they function, both PGK isoforms are cyanobacterial in origin. Apparently the cyanobacterial PGK gene was duplicated during plant evolution, with one 